EPJ Web of Conferences 82, 01002 (2015)

DOI: $10.1051 /$ epjconf/20158201002

(C) Owned by the authors, published by EDP Sciences, 2015

\title{
Water droplet deformation under the motion in gas area with subsonic velocities
}

\author{
Pavel A. Kuibin ${ }^{1,2}$ and Pavel A. Strizhak ${ }^{3, a}$ \\ ${ }^{1}$ Novosibirsk State University, 630090 Novosibirsk, Russia \\ ${ }^{2}$ Kutateladze Institute of Thermophysics, 630090 Novosibirsk, Russia \\ ${ }^{3}$ National Research Tomsk Polytechnic University, 634050 Tomsk, Russia
}

\begin{abstract}
The experimental investigation of water droplet deformation (characteristic dimensions $3 \div 6 \mathrm{~mm}$ ) when moving through the gas area (air) with $1 \div 5 \mathrm{~m} / \mathrm{s}$ velocities was carried out. The high-speed (delay time between the frames is less than $100 \mathrm{~ns}$ ) tools of cross-correlation videorecording were used. A cyclic change nature of the droplet shapes (from close to spherical to conditionally ellipsoidal) was found. The characteristic times of "deformation cycles" and the maximal deviations of droplet dimensions relative to initial dimensions were determined. The influence of droplet velocities and its dimensions on the deformation characteristics was determined.
\end{abstract}

\section{Introduction}

The liquid droplet shape variability under its motion through the gas area (in particular, during free falling in the air) was experimentally determined sufficiently long ago [1, 2].

The advent (during the last decade) of video- and cross-correlation cameras with a high frame sampling and determination procedures of particle and droplet dimensions predetermined the investigations of deformation, crushing and coagulation mechanisms of droplets in various gas areas [3-5]. The numerical value determination (using the high-speed video- and cross-correlation cameras) of main droplet deformation process characteristics for the most typical and widely used in various applications liquid (water) when moving through the gas area with low (less than $5 \mathrm{~m} / \mathrm{s}$ ) speeds is of interest. The purpose of this article is the experimental determination of numerical parameter values and water droplet configuration change mechanism when moving through the gas area.

\section{Experimental setup and procedure}

According to main measuring instruments the setup is similar to the setup used during the experimentation concerning the evaporation processes of single water droplets and water droplet group in high temperature (more $1000 \mathrm{~K}$ ) gas area [6-8] using the optical diagnostic methods of two-phase

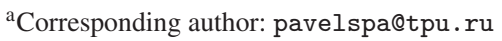

This is an Open Access article distributed under the terms of the Creative Commons Attribution License 4.0, which permits unrestricted use, distribution, and reproduction in any medium, provided the original work is properly cited. 


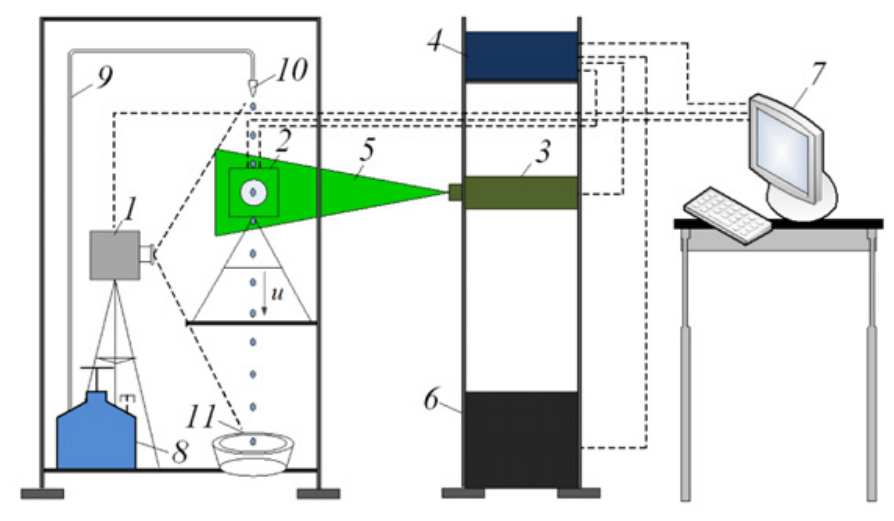

Figure 1. A schematic illustration of experimental setup: 1 - high-speed video camera; 2 - cross-correlation camera; 3 - double pulsed solid-state laser; 4 - synchronizer of personal computer (PC), cross-correlation camera and laser; 5 - light "pulse"; 6- laser emission generator; 7 - PC; 8 - vessel with a water; 9 - water feed channel; 10 - doser; 11 - catcher.

gas-vapor-liquid flows ("Particle Image Velocimetry" (PIV) [9] and "Interferometric Particle Imaging" (IPI) [10]).

The following routine of experiments (Fig. 1) was developed. The water from vessel 8 flows through channel 9 at the inlet of doser 10. The single droplets flying through the recording area in the air to catcher 11 outflow from doser 10 in accordance with predetermined initial dimensions $d_{0}$ and velocity $u_{0}$. The droplet motion process is recorded by a video camera $l$ (with frequency -10000 frames per second). So, it is carried out not less than 10 experiments for a single dimension and droplet velocity under the other fixed conditions. The regions with characteristic droplet configuration variations are selected after processing of videograms on a personal computer (PC) 7. The time intervals $t_{d}$, when the droplets finish the full "deformation cycle", i.e. sequentially twice take shape close to identical, are fixed. The videogram analysis of experiments carried out carry inference that a "deformational cycle" term can be used for a droplet shape change process under the conditions considered. The distance between a doser 10 and catcher 11 is divided into the group of regions characterizing the corresponding "deformational cycles" during the videogram processing, and the "repeatability" analysis of measuring results (both with respect to time $t_{\mathrm{d}}$ and characteristic length $s_{\mathrm{d}}$ ) is carried out.

\section{Results and discussion}

According to the results of experiments carried out, inference should be drawn that sufficiently intense droplet deformation takes place during the flight. Thus, typical videogram frames of experiments with pictures of a deforming water droplet in the process of its motion are shown in Fig. 2.

It should be pointed out that the droplet configurations during one of characteristic interim "deformation cycles" $\left(t_{\mathrm{d}}=0.021 \mathrm{~s}, s_{\mathrm{d}}=57.2 \mathrm{~mm}\right)$ during the passage of distance approximately $0.5 \mathrm{~m}$ from doser 10 in the direction of catcher 11 (Fig. 1) are presented in Fig. 2. The experiments carried out showed (Fig. 2) that water droplets change the shape cyclically (from spherical to conditionally ellipsoidal).

Figures 3 and 4 show the changes in the water droplet deformation $\left(\Delta_{x}, \Delta_{y}\right.$ and $\left.\Delta_{\max }\right)$ characteristics (at $u=1.5 \mathrm{~m} / \mathrm{s}, d_{0}=4 \mathrm{~mm}$ ) during several "deformational cycles" with corresponding times $t_{\mathrm{d}}$.

It may be noted the continuous (Fig. 3,4) variation of droplet shape during a sufficiently small time interval $\left(t_{\mathrm{d}} \ll 1 \mathrm{~s}\right)$. This result, in particular, carries inference that the approaches based on the guesses about the permanence of its shape (sphere, ellipsoid, disk or, for example, prolate cylinder) 


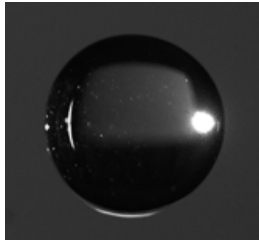

$a$

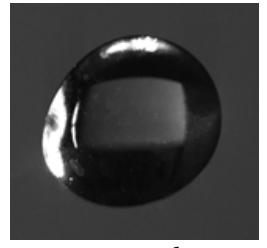

$b$

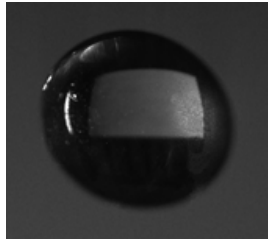

C

Figure 2. Pictures of water droplets at $u=3 \mathrm{~m} / \mathrm{s}, d_{0}=3 \mathrm{~mm}$ during a time interval $0<t<t_{\mathrm{d}}: a-t=0 \mathrm{~s}$, $b-t=t_{\mathrm{d}} / 6, c-t=t_{\mathrm{d}} / 2$.

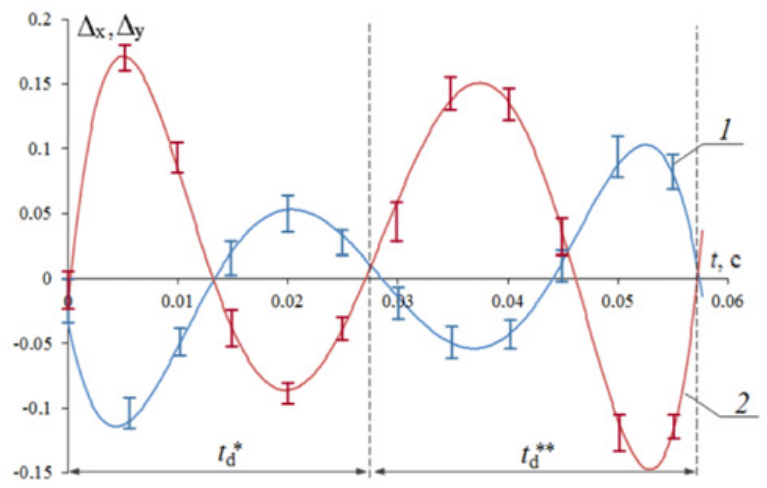

Figure 3. $\Delta_{x}$ and $\Delta_{y}$ values at $u=1.5 \mathrm{~m} / \mathrm{s}$ and $d_{0}=4 \mathrm{~mm}$ during several characteristic "deformation cycles": $1-$ $\Delta_{x}, 2-\Delta_{y}, *$ - first "deformation cycle" under consideration, ** - second "deformation cycle" under consideration.

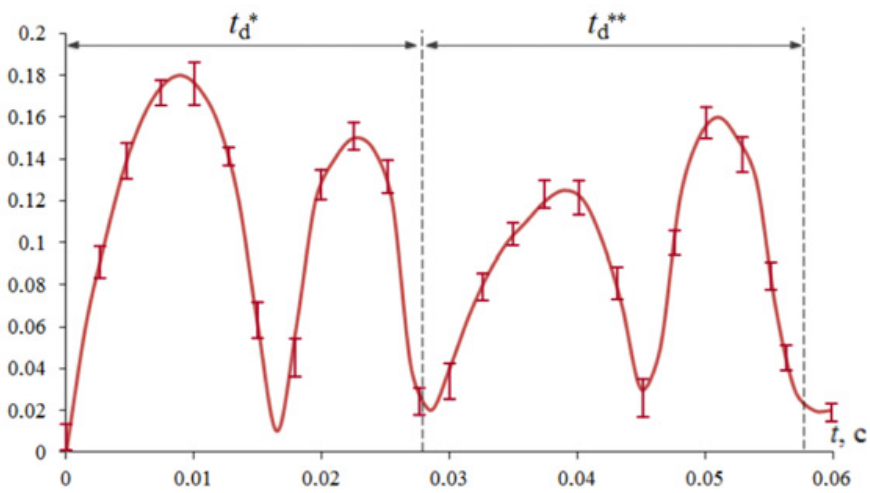

Figure 4. $\Delta_{\max }$ values at $u=1.5 \mathrm{~m} / \mathrm{s}$ and $d_{0}=4 \mathrm{~mm}$ during several characteristic "deformation cycles": * next "deformation cycle".

are not exactly substantiated to use in the numerical simulation of liquid droplet motion processes. At that it can be assumed that characteristic times $t_{\mathrm{d}}$ will be less than found (in the experiments under consideration) times under the conditions of intense phase transformations (for example, when moving through the high temperature gas area [6-8]), and the deformation effect will perform a more important role. 
Figure 3 shows the asymmetry of changes in droplet dimensions in two coordinates of videogram planes $\left(\Delta_{x} \neq \Delta_{y}\right)$. It may be concluded concerning the change in droplet dimensions in all three coordinates and the expediency of account of this effect, when physical and mathematical models of such processes are developed. Obtained according to the experiment results asymmetry and variety of droplet shapes during its motion are reason for a conclusion about three-dimensional droplet deformation. $\Delta_{\max }$ values in the experiments carried out (under the conditions of $u$ and $d_{0}$ variation in the range of $1 \div 5 \mathrm{~m} / \mathrm{s}$ and $3 \div 6 \mathrm{~mm}$ ) were less than 0.25 . According to confidential determination interval (which are presented in Fig. 3 and 4) of droplet dimensions in the experiments, inference should be drawn that the random inaccuracy of $d_{\mathrm{x}}, d_{\mathrm{y}}$ and $d_{\max }$ determination are sufficiently less than values of these parameters.

It has been established that the growth of droplet dimensions leads to the insignificant (less than $7 \%$ ) decrease of maximal deviations $\left(\Delta_{\max }\right)$ of its shapes from initial (close to spherical) in consequence of deformation during processing of $\Delta_{\mathrm{x}}=f(t), \Delta_{\mathrm{y}}=f(t)$ and $\Delta_{\max }=f(t)$ dependencies for the experiments with $u=15 \mathrm{~m} / \mathrm{s}$ and $d_{0}=36 \mathrm{~mm}$. The increase of $u$ droplet motion speeds in the range under consideration leads to $\Delta_{\mathrm{x}}, \Delta_{\mathrm{y}}$ and $\Delta_{\max }$ growth of $12 \div 17 \%$ (the amplitudes characterizing $d_{\mathrm{x}}, d_{\mathrm{y}}$ and $d_{\max }$ change are increased). The response time increase of deformational droplet motion by means of droplet mass growth can be considered as the main reason of $\Delta_{\max }$ decrease under the condition of $d_{0}$ increase.

Found according to experiment results regularities of the processes under study illustrate not only the influence of dimensions and water droplet motion speeds on the characteristics of droplet configuration change. The experimental results are reason for the further development of liquid droplet motion models in the gas area (including the high temperature gas area - more than $1000 \mathrm{~K}$ ).

\section{Conclusions}

1. Intense cyclic deformation with short characteristic times of "deformational cycles" $\left(t_{\mathrm{d}} \ll 1 \mathrm{~s}\right)$ takes place during the water droplet motion through the gas area under the action of gravity with subsonic (less than $5 \mathrm{~m} / \mathrm{s}$ ) velocities. $2 . \Delta_{\max }$ amplitudes grow by $12 \div 17 \%$ in consequence of $u$ velocity growth under the conditions of water droplets passage (in the air) of distances which exceed $s_{\mathrm{d}}$ values for one of initial "deformational cycles" by several dozens of times. 3. The usage of models taking into account the change of aerodynamic drag factor in time in accordance with possible "deformation cycles" is appropriate in solving problems of hydrodynamics and heat and mass transfer in gas-droplet flows.

The investigation was performed by Russian Foundation for Basic Research (project 14-38-50004).

\section{References}

[1] Ya.E. Geguzin, Kaplya (Science, Moskow, 1973) [in Russian]

[2] A. Wierzba, Exp. in Fluids 1, 9 (1990)

[3] A.K. Flock, D.R. Guildenbecher, J. Chen, P.E. Sojka, and H.J. Bauer, Int. Journ. of Multiph. Flow. 47 (2012)

[4] J.E. Sprittles and Y.D. Shikhmurzaev, Phys. of Fluids 24 (2012)

[5] R.N. El-Sayed, M. Albeirutty, and Y. Takata, Int. Journ. of Therm. Scien. 79 (2014)

[6] R.S. Volkov, O.V. Vysokomornaya, G.V. Kuznetsov, and P.A. Strizhak, Journ. of Eng. Phys. and Thermophys. 6, 86 (2014)

[7] R.S. Volkov, G.V. Kuznetsov, and P.A. Strizhak, Journ. of Eng. Phys. and Thermophys. 2, 87 (2014)

[8] R.S. Volkov, G.V. Kuznetsov, and P.A. Strizhak, Tech. Phys. 7, 59 (2014)

[9] J. Westerweel, Meas. Scien. and Techn. 8 (1997)

[10] N. Damaschke, H. Nobach, and C. Tropea, Exp. in Fluids 2, 32 (2002) 